\title{
Stereoselective Oxidation and Glucuronidation of Carvedilol in Human Liver and Intestinal Microsomes
}

\author{
Kazuya Ishida, Shigehiro TAIRA, Hiroki Morishita, Yuichiro Kayano, Masato Taguchi, and \\ Yukiya HAshimoto* \\ Graduate School of Medicine and Pharmaceutical Sciences, University of Toyama; 2630 Sugitani, Toyama 930-0194, \\ Japan. Received February 8, 2008; accepted March 22, 2008; published online March 27, 2008
}

\begin{abstract}
The aim of the present study was to investigate the mechanism for the stereoselective presystemic clearance of carvedilol. We examined the oxidation and glucuronidation of carvedilol in human liver microsomes (HLM) and human intestinal microsomes (HIM). The oxidation of carvedilol in HLM and HIM was evaluated in the presence of NADPH, whereas glucuronidation was evaluated in the presence of UDP-glucuronic acid. Oxidation of $\boldsymbol{S}$-carvedilol in HLM and HIM was greater than that of $\boldsymbol{R}$-carvedilol. In addition, the oxidation of $\boldsymbol{R}$-carvedilol in HLM was inhibited by quinidine, whereas that of $S$-carvedilol was inhibited by both quinidine and furafylline. On the other hand, $R$ - and $S$-carvedilol oxidation in HIM was inhibited by ketoconazole. Glucuronidation of $S$ carvedilol in HLM and HIM was also higher than that of $R$-carvedilol. These results suggested that cytochrome P450 (CYP) 2D6 and CYP1A2 are involved in the stereoselective oxidation of carvedilol in the liver, that CYP3A4 is involved in intestinal oxidation, and that glucuronidation in the liver and intestine is at least partly responsible for stereoselective presystemic clearance.
\end{abstract}

Key words carvedilol; stereoselective metabolism; liver; intestine

Carvedilol is a $\beta$-adrenoceptor antagonist that has been clinically used to treat chronic heart failure as well as hypertension, angina pectoris, and cardiac arrhythmias. ${ }^{1-4)}$ Carvedilol is highly lipophilic and is absorbed rapidly from the gastrointestinal tract after oral administration. Orally administered carvedilol undergoes stereoselective first-pass metabolism, and the blood concentration of the $R$-enantiomer with very low $\beta$-blocking activity is approximately 2 -fold higher than that of the $S$-enantiomer with high $\beta$-blocking activity. ${ }^{5,6)}$ Both enantiomers are mostly eliminated by hepatic metabolism, with renal excretion accounting for only $0.3 \%$ of the administered dose. ${ }^{7}$ Carvedilol is metabolized extensively via aliphatic side-chain oxidation, aromatic ring oxidation, and conjugation pathways. ${ }^{8)}$ Oldham and Clarke reported that oxidative activity for carvedilol is observed in cytochrome P450 (CYP) 2D6, 2C9, 3A4, and 1A2. ${ }^{9)}$ In addition, Ohno et al. reported that UDP-glucuronosyltransferase (UGT) 2B7, 2B4, and 1A1 are capable of catalyzing the glucuronidation of carvedilol. ${ }^{10)}$ In 2007, Takekuma et al. reported that glucuronidation of racemic carvedilol in human liver microsomes (HLM) was stereoselective for the $S$-enantiomer, and that UGT2B7 may be involved in the stereoselective glucuronidation of carvedilol in the human liver. ${ }^{11)} \mathrm{How}-$ ever, it is still unclear whether the oxidation of carvedilol in the liver is stereoselective for the $S$-enantiomer, and also unclear which CYP isoform is primarily responsible for the oxidation of $R$ - and $S$-carvedilol in the liver. Furthermore, although CYP3A4 and several UGT isoforms are expressed in intestinal epithelial cells, it is unknown whether the intestine also plays a role in the first-pass presystemic metabolism of carvedilol. ${ }^{12-14)}$

In the present study, to investigate the mechanism for the stereoselective presystemic clearance of carvedilol, we evaluated the oxidation and glucuronidation of carvedilol in HLM and human intestinal microsomes (HIM). The oxidation of carvedilol in HLM and HIM was evaluated in the presence of NADPH, whereas glucuronidation was evaluated in the pres- ence of UDP-glucuronic acid (UDPGA).

\section{MATERIALS AND METHODS}

Materials Pooled HLM and HIM were purchased from BD Biosciences (Woburn, MA, U.S.A.). Carvedilol was kindly supplied by Daiichi Pharmaceutical (Tokyo, Japan). UDPGA trisodium salt, furafylline, sulfaphenazole, and quinidine hydrochloride were purchased from Sigma-Aldrich (St. Louis, MO, U.S.A.). Ketoconazole was obtained from Wako Pure Chemicals (Osaka, Japan). $\left[{ }^{14} \mathrm{C}\right]$ UDPGA (180.3 $\mathrm{mCi} / \mathrm{mmol}$ ) was purchased from PerkinElmer Life and Analytical Sciences (Boston, MA, U.S.A.). TLC plates $(20 \times 20$ $\mathrm{cm}$ Silica gel 60 F254, with a concentration zone $20 \times 2.5$ $\mathrm{cm}$ ) were purchased from Merck (Whitehouse Station, NJ, U.S.A.). All other chemicals were of the highest purity available.

Preparation of Rat Liver Microsomes (RLM) Male Wistar rats $(240-260 \mathrm{~g})$ were purchased from Japan SLC Inc. (Hamamatsu, Japan), and the animal experiments were performed in accordance with the Guidelines for Animal Experiments of the University of Toyama. RLM were prepared as previously reported. ${ }^{15)}$ Briefly, livers were removed from rats and homogenized in 3 volumes of the original liver weight of ice-cold $1.15 \%$ potassium chloride using a PotterElvehjem homogenizer. The homogenate was centrifuged at $9000 \boldsymbol{g}$ for $20 \mathrm{~min}$, and the supernatant was transferred and centrifuged again at $105000 \mathrm{~g}$ for $60 \mathrm{~min}$. The pellet was resuspended in 0.3 volumes of the original liver weight of icecold $1.15 \%$ potassium chloride. The microsomal suspensions were stored at $-85^{\circ} \mathrm{C}$ until use.

Oxidation of $\boldsymbol{R}$ - and $\boldsymbol{S}$-Carvedilol in RLM, HLM, and HIM Oxidation of $R$ - and $S$-carvedilol in RLM, HLM, and HIM were evaluated in the presence of NADPH. The incubation mixture (final volume $100 \mu \mathrm{l}$ ) consisted of $1 \mu \mathrm{M}$ racemic carvedilol, $1.0 \mathrm{mg} / \mathrm{ml}$ microsomal proteins, $2 \mathrm{~mm}$ NADPH, and $50 \mathrm{~mm}$ potassium phosphate buffer ( $\mathrm{pH}$ 7.4). Carvedilol 
was dissolved in a mixture of dimethylformamide and acetonitrile, and the final concentrations of dimethylformamide and acetonitrile in the mixture were $0.05 \%$ and $0.02 \%(\mathrm{v} / \mathrm{v})$, respectively. ${ }^{12)}$ The reaction was allowed to run for $15 \mathrm{~min}$ (RLM and HLM) or $40 \mathrm{~min}$ (HIM) at $37^{\circ} \mathrm{C}$. The metabolized amount of $R$ - and $S$-carvedilol was calculated by subtracting the amount remaining in the sample from the amount applied. We further evaluated the effect of furafylline $(25 \mu \mathrm{M})$, sulfaphenazole $(10 \mu \mathrm{M})$, quinidine $(1 \mu \mathrm{M})$, and ketoconazole $(1 \mu \mathrm{M})$ on the oxidation of carvedilol in HLM and HIM. ${ }^{9,16)}$

Glucuronidation of $\boldsymbol{R}$ - and $\boldsymbol{S}$-Carvedilol in RLM, HLM, and HIM Glucuronidation of $R$ - and $S$-carvedilol in RLM, HLM, and HIM were evaluated in the presence of UDPGA. The incubation mixture (final volume $100 \mu \mathrm{l}$ ) consisted of $1 \mu \mathrm{M}$ racemic carvedilol, $1.0 \mathrm{mg} / \mathrm{ml}$ microsomal proteins, $10 \mathrm{~mm} \mathrm{MgCl}_{2}, 2 \mathrm{~mm}$ UDPGA, $25 \mu \mathrm{g} / \mathrm{ml}$ alamethicin, and $50 \mathrm{~mm}$ Tris- $\mathrm{HCl}$ buffer (pH 7.4). The reaction was allowed to run for $45 \mathrm{~min}$ (RLM and HLM) or $120 \mathrm{~min}$ (HIM) at $37^{\circ} \mathrm{C}$. The metabolized amount of $R$ - and $S$ carvedilol was calculated by subtracting the amount remaining in the sample from the amount applied.

Assay of Carvedilol The amount of carvedilol in the samples was measured using chiral high performance liquid chromatography (HPLC) as described previously with a minor modification. ${ }^{6,12)}$ Briefly, after alkalization in $3 \mathrm{ml}$ of $0.1 \mathrm{~m}$ Britton-Robinson buffer $(\mathrm{pH} 8.5)$, samples $(0.1 \mathrm{ml})$ were extracted with $5 \mathrm{ml}$ of diethylether. The organic phase was transferred and evaporated dry in a water bath at $45^{\circ} \mathrm{C}$. The residue was dissolved in $500 \mu \mathrm{l}$ of mobile phase, and $70 \mu \mathrm{l}$ was injected into the HPLC column. The HPLC system was a Shimadzu LC-10AS (Kyoto, Japan). Separation was achieved with a chiral stationary phase column (CHIRALPAC AD-H: $5 \mu \mathrm{m}$ particle size, $2 \mathrm{~mm}$ i.d. $\times 25 \mathrm{~cm}$; Daicel Chemical Industries, Tokyo, Japan). The temperature of the column oven was set at $40^{\circ} \mathrm{C}$. The mobile phase consisted of $73 \%$ hexane, $27 \%$ isopropanol, and $0.1 \%(\mathrm{v} / \mathrm{v})$ diethylamine, and the flow rate was $0.3 \mathrm{ml} / \mathrm{min}$. The peaks were monitored at an excitation wavelength of $284 \mathrm{~nm}$ and an emission wavelength of $343 \mathrm{~nm}$ (Shimadzu RF-10A). The detection limit for each enantiomer was $0.5 \mathrm{nM}$ for the concentration in the samples. The coefficient of inter-day variation for the assay of $R$ - and $S$-carvedilol was $13.1 \%$ and $12.7 \%$, respectively, at the concentration of $500 \mathrm{~nm}$.

Formation of Carvedilol Glucuronides in RLM, HLM, and HIM Formation of carvedilol glucuronides in RLM, HLM, and HIM were evaluated as described by Ohno et $a l .{ }^{10)}$ The incubation mixture (final volume $100 \mu \mathrm{l}$ ) consisted of $100 \mu \mathrm{M}$ racemic carvedilol, $1.0 \mathrm{mg} / \mathrm{ml}$ microsomal proteins, $10 \mathrm{~mm} \mathrm{MgCl}_{2}, 2 \mathrm{~mm}$ UDPGA containing $0.3 \mu \mathrm{Ci}$ $\left[{ }^{14} \mathrm{C}\right]$ UDPGA, $25 \mu \mathrm{g} / \mathrm{ml}$ alamethicin, and $50 \mathrm{~mm}$ Tris $-\mathrm{HCl}$ buffer ( $\mathrm{pH} 7.4)$. Carvedilol was dissolved in dimethylsulfoxide $/ 0.05 \mathrm{~N} \mathrm{NaOH}(50: 50, \mathrm{v} / \mathrm{v})$, and the final concentration of dimethylsulfoxide in the incubation mixture was $1.5 \%(\mathrm{v} / \mathrm{v})$. The reaction was allowed to run for $60 \mathrm{~min}$ at $37^{\circ} \mathrm{C}$, and was terminated by the addition of $100 \mu \mathrm{l}$ of methanol. Centrifugation at $2380 \boldsymbol{g}$ for $20 \mathrm{~min}$ at $4{ }^{\circ} \mathrm{C}$ was performed to remove proteins. A $20-\mu \mathrm{l}$ aliquot of the supernatant was spotted directly onto a TLC plate and subjected to chromatography in a solvent containing chloroform/methanol/ethanol/acetic $\mathrm{acid} /$ water $(10: 4: 2: 1: 1, \mathrm{v} / \mathrm{v})$. The dried plate was exposed for $72 \mathrm{~h}$ to a BAS imaging plate, and spots of carvedilol glu- curonide were visualized using a BAS-5000 radioisotope imaging analyzer (Fujifilm, Tokyo, Japan).

Data Analysis Values are expressed as the mean \pm S.E. Differences between two groups were evaluated with the Wilcoxon signed-rank test. Multiple comparisons were performed using Scheffé's test following one-way ANOVA provided that the variances of the groups were similar. If this was not the case, a Scheffé-type test was applied following Kruskal-Wallis analysis. $p<0.05$ was considered to be statistically significant.

\section{RESULTS AND DISCUSSION}

Oxidation of $\boldsymbol{R}$ - and $\boldsymbol{S}$-Carvedilol in HLM and HIM We first evaluated the oxidation of $R$ - and $S$-carvedilol in RLM (as a control), HLM, and HIM at the microsomal protein concentration of $1 \mathrm{mg} / \mathrm{ml}$ and at the racemic drug concentration of $1 \mu \mathrm{M}$. In addition, the concentration of carvedilol was much lower than the apparent $K_{\mathrm{m}}$ values $(>20 \mu \mathrm{M})$ for the oxidative metabolites of the drug by HLM. ${ }^{9)}$ Therefore, the oxidative metabolism of carvedilol in HLM was not thought to be saturated in the present experimental condition. Figure 1 shows the percentage of $R$ - and $S$ carvedilol oxidized following 15-, 15-, and 40-min incubation in RLM, HLM, and HIM, respectively. The oxidation activity of HLM for $R$ - and $S$-carvedilol was lower than that of RLM (Figs. 1A, B). The percentage of $S$-carvedilol metabolized in HLM (74.3\%) was 1.7-fold higher than that of $R$ carvedilol (43.8\%). Carvedilol was less oxidized in HIM than in HLM, even though the reaction time was longer for HIM than for HLM (Figs. 1B, C). However, the percentage of $S$-carvedilol metabolized in HIM $(53.4 \%)$ was also 1.5 fold higher than that of $R$-carvedilol $(35.7 \%)$. These findings indicated that the oxidation activity of the human liver and intestine is lower than that of the rat liver, but that oxidation of carvedilol not only in the human liver but also in the human intestine is stereoselective for $S$-enantiomer. To our knowledge, this is the first report of stereoselective oxidation of carvedilol in the human intestine.

Contribution of CYP Isoforms to the Stereoselective Oxidation of Carvedilol in HLM and HIM To identify the CYP isoforms responsible for the stereoselective oxidation of carvedilol in HLM and HIM, we further examined the effect of CYP-specific inhibitors on the oxidation of $R$ - and

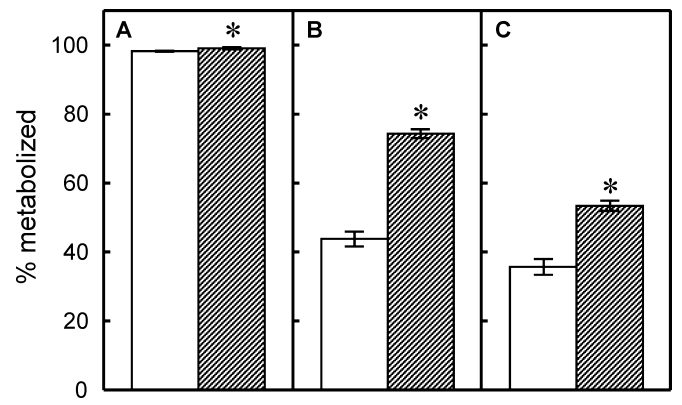

Fig. 1. Stereoselective Oxidation of Carvedilol in RLM (A), HLM (B), and HIM $(\mathrm{C})$

Microsomes were incubated with $1 \mu \mathrm{M}$ racemic carvedilol for $15 \mathrm{~min}$ (RLM and HLM) or $40 \mathrm{~min}$ (HIM) in the presence of $2 \mathrm{~mm}$ NADPH. Open and hatched columns indicate $R$-carvedilol and $S$-carvedilol, respectively. Each column represents the mean \pm S.E. for 7 experiments. $* p<0.05$ compared with $R$-carvedilol. 
$S$-carvedilol in HLM and HIM by applying $25 \mu \mathrm{M}$ furafylline (an inhibitor of CYP1A2), $10 \mu \mathrm{M}$ sulfaphenazole (an inhibitor of CYP2C9), $1 \mu \mathrm{M}$ quinidine (an inhibitor of CYP2D6), and $1 \mu \mathrm{M}$ ketoconazole (an inhibitor of CYP3A4). In addition, the concentrations of CYP-specific inhibitors were determined according to the reports of Oldham and Clarke $^{9)}$ and Newton et al. ${ }^{16)}$ Table 1 shows the oxidation activities of HLM and HIM for $R$ - and $S$-carvedilol in the presence of inhibitors. The oxidation of $R$-carvedilol in HLM was significantly inhibited by quinidine (29.7\% of control), whereas that of $S$-carvedilol was markedly inhibited by both quinidine and furafylline $(74.8 \%$ and $65.5 \%$ of control, respectively). In contrast, the oxidation of $R$ - and $S$-carvedilol in HIM was significantly inhibited by ketoconazole $(28.1 \%$ and $30.4 \%$ of control, respectively). These findings suggested that CYP2D6 and CYP1A2 are primarily responsible for the stereoselective oxidation of carvedilol in the human liver, and that CYP3A4 in the human intestine is also responsible for presystemic clearance.

Glucuronidation of $\boldsymbol{R}$ - and $\boldsymbol{S}$-Carvedilol in HLM and HIM We also evaluated the glucuronidation of $R$ - and $S$ carvedilol in RLM (as a control), HLM, and HIM at the microsomal protein concentration of $1 \mathrm{mg} / \mathrm{ml}$ and at the racemic

Table 1. Effect of CYP-Specific Inhibitors on the Stereoselective Oxidation of Carvedilol in HLM and HIM

\begin{tabular}{|c|c|c|}
\hline & \multicolumn{2}{|c|}{$\%$ of control } \\
\hline & $R$-Carvedilol & $S$-Carvedilol \\
\hline \multicolumn{3}{|l|}{ HLM } \\
\hline Control & $100 \pm 2.0^{a)}$ & $100 \pm 0.7^{b)}$ \\
\hline$+25 \mu \mathrm{M}$ furafylline & $85.1 \pm 4.3$ & $65.5 \pm 1.7 *$ \\
\hline$+10 \mu \mathrm{M}$ sulfaphenazole & $81.7 \pm 4.9$ & $87.9 \pm 1.7 *$ \\
\hline$+1 \mu \mathrm{M}$ quinidine & $29.7 \pm 9.5^{*}$ & $74.8 \pm 2.9^{*}$ \\
\hline$+1 \mu \mathrm{M}$ ketoconazole & $85.0 \pm 5.8$ & $87.8 \pm 1.8 *$ \\
\hline \multicolumn{3}{|l|}{ HIM } \\
\hline Control & $100 \pm 7.0^{c)}$ & $100 \pm 4.0^{d)}$ \\
\hline$+25 \mu \mathrm{M}$ furafylline & $91.6 \pm 14.4$ & $95.9 \pm 9.1$ \\
\hline$+10 \mu \mathrm{m}$ sulfaphenazole & $83.2 \pm 13.3$ & $88.4 \pm 8.0$ \\
\hline$+1 \mu \mathrm{M}$ quinidine & $81.2 \pm 2.1$ & $89.8 \pm 1.5$ \\
\hline$+1 \mu \mathrm{M}$ ketoconazole & $28.1 \pm 13.6^{*}$ & $30.4 \pm 9.6^{*}$ \\
\hline
\end{tabular}

Microsomes were incubated with $1 \mu \mathrm{M}$ racemic carvedilol for $15 \min$ (HLM) and $40 \mathrm{~min}$ (HIM) in the presence of $2 \mathrm{~mm}$ NADPH and $1-25 \mu \mathrm{M}$ inhibitors. Values are expressed as the mean \pm S.E. for $6-8$ (HLM) and 3 (HIM) experiments. a) $37.1 \%$ of dose. b) $66.1 \%$ of dose. c) $39.2 \%$ of dose. d) $52.1 \%$ of dose. $* p<0.05$ compared with the control.

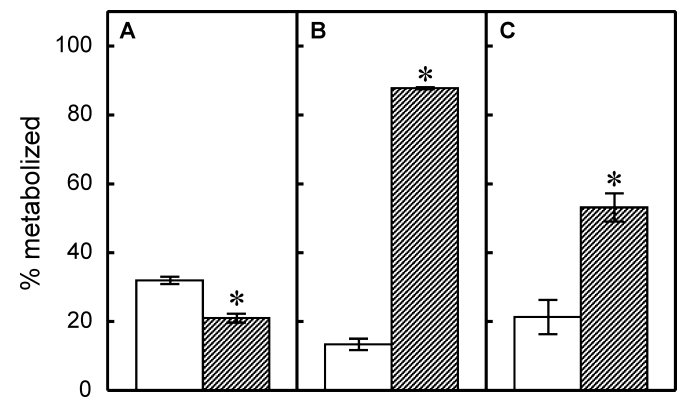

Fig. 2. Stereoselective Glucuronidation of Carvedilol in RLM (A), HLM (B), and HIM (C)

Microsomes were incubated with $1 \mu \mathrm{M}$ racemic carvedilol for $45 \mathrm{~min}$ (RLM and HLM) or $120 \mathrm{~min}$ (HIM) in the presence of $2 \mathrm{~mm}$ UDPGA. Open and hatched columns indicate $R$-carvedilol and $S$-carvedilol, respectively. Each column represents the mean \pm S.E. for 7 experiments. $* p<0.05$ compared with $R$-carvedilol. drug concentration of $1 \mu \mathrm{M}$. Figure 2 shows the percentage of $R$ - and $S$-carvedilol metabolized following 45-, 45-, and 120min incubation in RLM, HLM, and HIM, respectively. The glucuronidation activity of HLM for $R$-carvedilol was lower than that of RLM, whereas the glucuronidation activity of HLM for $S$-carvedilol was higher than that of RLM (Figs. $2 \mathrm{~A}, \mathrm{~B})$. In addition, the percentage of $S$-carvedilol metabolized in RLM $(21.0 \%)$ was lower than that of $R$-carvedilol $(32.0 \%)$, whereas the percentage of $S$-carvedilol metabolized in HLM (87.8\%) was 6.5-fold higher than that of $R$ carvedilol (13.4\%). Carvedilol glucuronidation in HIM was similar to or less than in HLM, even though the reaction time was longer for HIM than for HLM (Figs. 2B, C). However, the percentage of $S$-carvedilol metabolized in HIM $(53.1 \%)$ was also 2.5 -fold higher than that of $R$-carvedilol $(21.3 \%)$. These findings indicated that glucuronidation of carvedilol in the human liver and intestine is stereoselective for the $S$ enantiomer, and is at least partly responsible for stereoselective presystemic clearance. To our knowledge, this is the first report of stereoselective glucuronidation of carvedilol in the human intestine.

Formation of Carvedilol Glucuronides in HIM We finally evaluated the formation of carvedilol glucuronides in RLM (as a control), HLM (as a control), and HIM using TLC analysis as described by Ohno et al. ${ }^{10)}$ Figure 3 shows spots of carvedilol glucuronides formed by RLM, HLM, and HIM following 60 -min incubation at the microsomal protein concentration of $1 \mathrm{mg} / \mathrm{ml}$ and at the racemic drug concentration of $100 \mu \mathrm{M}$. G1 and G2 were generated by RLM and HLM. The amount of G1 formed by RLM was less than that of $\mathrm{G} 2$ (G1/G2 ratio of 0.65$)$, whereas the amount of $\mathrm{G} 1$ formed by HLM was more than that of $\mathrm{G} 2(\mathrm{G} 1 / \mathrm{G} 2$ ratio of 2.7). These findings in conjunction with the results shown in Figs. 2A and B suggested that G1 and G2 were derived from $S$ - and $R$-carvedilol, respectively. G1 and G2 were both generated by HIM, although the amounts were much less than those formed by RLM and HLM (Fig. 3). Ohno et al. reported that UGT2B7 and 2B4 were responsible for the formation of $\mathrm{G} 1$, and that UGT1A1 and $2 \mathrm{~B} 4$ were responsible for the formation of $\mathrm{G} 2 .^{10)}$ Therefore, the present findings suggested that UGT2B7 and also UGT1A1 (and/or UGT2B4) are plausible candidates for the glucuronidation of carvedilol in the human intestine. Further studies are necessary to identify the UGT isoform(s) responsible for the glu-

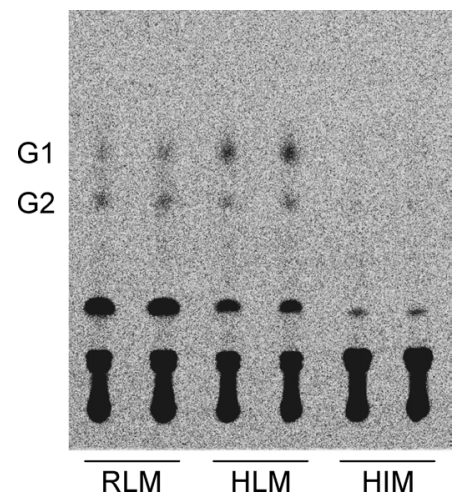

Fig. 3. TLC Analysis of Carvedilol Glucuronides

Microsomes were incubated with $100 \mu \mathrm{M}$ racemic carvedilol for $60 \mathrm{~min}$ in the presence of $2 \mathrm{~mm}$ UDPGA containing $0.3 \mu \mathrm{Ci}\left[{ }^{14} \mathrm{C}\right] \mathrm{UDPGA}$. 
curonidation of carvedilol in the human intestine.

In conclusion, the present findings indicated that not only glucuronidation but also oxidation of carvedilol in the human liver is stereoselective for the $S$-enantiomer, and that CYP2D6 and CYP1A2 in the human liver are mainly responsible for oxidation. Furthermore, both oxidation and glucuronidation of carvedilol in HIM were also stereoselective for the $S$-enantiomer, suggesting that the human intestine as well as the liver is involved in first-pass metabolism. In addition, CYP3A4 in the human intestine may be involved in the oxidation of carvedilol, and the UGT2B and UGT1A subfamilies may be responsible for glucuronidation in the intestine.

Acknowledgement This work was supported in part by a Grant-in-Aid for Scientific Research from the Japan Society for the Promotion of Sciences (JSPS).

\section{REFERENCES}

1) Packer M., Bristow M. R., Cohn J. N., Colucci W. S., Fowler M. B., Gilbert E. M., Shusterman N. H., N. Engl. J. Med., 334, 1349-1355 (1996).

2) Eggertsen R., Sivertsson R., Andren L., Hansson L., J. Cardiovasc. Pharmacol., 10 (Suppl. 11), S97-S100 (1987).
3) Nagele H., Bohlmann M., Eck U., Petersen B., Rodiger W., Eur. J. Heart Fail., 2, $71-79$ (2000).

4) Nahrendorf W., Rading A., Steinig G., van der Does R., Schlote A., J. Cardiovasc. Pharmacol., 19 (Suppl. 1), S114-S116 (1992).

5) Honda M., Nozawa T., Igarashi N., Inoue H., Arakawa R., Ogura Y., Okabe H., Taguchi M., Hashimoto Y., Biol. Pharm. Bull., 28, 14761479 (2005).

6) Honda M., Ogura Y., Toyoda W., Taguchi M., Nozawa T., Inoue H., Hashimoto Y., Biol. Pharm. Bull., 29, $772-778$ (2006).

7) Fujimaki M., Murakoshi Y., Hakusui H., J. Pharm. Sci., 79, 568-572 (1990).

8) Neugebauer G., Akpan W., von Mollendorff E., Neubert P., Reiff K., J. Cardiovasc. Pharmacol., 10 (Suppl. 11), S85-S88 (1987).

9) Oldham H. G., Clarke S. E., Drug Metab. Dispos., 25, 970—977 (1997).

10) Ohno A., Saito Y., Hanioka N., Jinno H., Saeki M., Ando M., Ozawa S., Sawada J., Drug Meab. Disops., 32, 235-239 (2004).

11) Takekuma Y., Takenaka T., Yamazaki K., Ueno K., Sugawara M., Biol. Pharm. Bull., 30, 2146-2153 (2007).

12) Ishida K., Honda M., Shimizu T., Taguchi M., Hashimoto Y., Biol. Pharm. Bull., 30, 1930-1933 (2007).

13) Paine M. F., Hart H. L., Ludington S. S., Haining R. L., Rettie A. E., Zeldin D. C., Drug Metab. Dispos., 34, 880-886 (2006).

14) Ritter J. K., Expert Opin. Drug Metab. Toxicol., 3, 93-107 (2007).

15) Okabe H., Higashi T., Ohta T., Hashimoto Y., Biol. Pharm. Bull., 27, 1422-1427 (2004).

16) Newton D. J., Wang R. W., Lu A. Y., Drug Metab. Dispos., 23, 154 158 (1995). 